MSC 2010: 70F25, 70E60, 70Q05

\title{
Inverse Dynamics-Based Motion Control of a Fluid-Actuated Rolling Robot
}

\author{
S. A. Tafrishi, Y. Bai, M. Svinin, E. Esmaeilzadeh, M. Yamamoto
}

In this paper, the rest-to-rest motion planning problem of a fluid-actuated spherical robot is studied. The robot is driven by moving a spherical mass within a circular fluid-filled pipe fixed internally to the spherical shell. A mathematical model of the robot is established and two inverse dynamics-based feed-forward control methods are proposed. They parameterize the motion of the outer shell or the internal moving mass as weighted Beta functions. The feasibility of the proposed feed-forward control schemes is verified under simulations.

Keywords: inverse dynamics, motion planning, spherical robots, fluid actuator

\section{Introduction}

Spherical robots have gained the attention of robotic researchers in the last decade. These robots can roll in different environments with a reliance on their symmetric bodies [1]. However, controlling these robots with the use of conventional actuators is a challenging research problem.

Received June 05, 2019

Accepted November 20, 2019

This research was supported, in part, by the Japan Science and Technology Agency, the JST Strategic International Collaborative Research Program, Project No. 18065977.

Seyed Amir Tafrishi

amir@ce.mech.kyushu-u.ac.jp

Motoji Yamamoto

yama@mech.kyushu-u.ac.jp

Faculty of Engineering, Kyushu University

744 Motooka, Nishi-ku, Fukuoka 819-0395, Japan

Yang Bai

yangbai@fc.ritsumei.ac.jp

Mikhail Svinin

svinin@fc.ritsumei.ac.jp

College of Information Science and Engineering, Ritsumeikan University

1-1-1 Nojihigashi, Kusatsu, Shiga 525-8577, Japan

Esmaeil Esmaeilzadeh

esmzadeh@tabrizu.ac.ir

Department of Mechanical Engineering, University of Tabriz

Bahman Blvd. 29, Tabriz 51666-14766, Iran

RUSSIAN JOURNAL OF NONLINEAR DYNAMICS, 2019, 15(4), 611-622 
Based on the actuating schemes, spherical robots can be classified into following types. They can work by rotating internal wheels, which was named the torque-reaction principle $[2,3]$. To control the robots based on the torque-reaction principle, different control strategies were developed $[4,5]$. The control of such robots can be regarded as manipulating an omnidirectional car inside a ball if the center of mass of the robot always stays in the lower hemisphere. Another actuation principle is changing the center of mass of the rolling body [6, 7]. This actuation principle can be realized by moving internal weights connected to rods. Rotating a mass within the sphere is another alternative way to actuate a rolling robot [8-11]. Trajectory tracking for pendulum-actuated robots was considered in $[4,12,13,25]$. The third actuation principle is based on the conservation of the angular momentum [14-17]. Note that the driving units of rolling robots can employ combined principles of actuation [10, 18]. Such rolling robots may have promising agility, but developing control strategies requires an insightful study of their dynamical models and understanding the correlation between the driving principles.

In our previous work, we introduced a novel fluid-actuated spherical robot [11, 19]. This robot employs the mass-imbalance principle based on rotating a spherical mass (core) inside circular pipes. To move the core, a fluid actuator creates pressure in the pipe. However, the dynamics-based control strategies for this robot have not been studied so far. In this paper, we establish and test under simulation the feed-forward motion planning strategies based on the direct specification of either the core or the sphere motion by properly selected time functions.

The paper is organized as follows. In Section 2, the dynamics of the rolling robot are derived. The inverse dynamics and feed-forward control strategies realizing rest-to-rest motion are analyzed in Section 3. Simulation results and the performance of the proposed control strategies are discussed in Section 4. Finally, conclusions are drawn in Section 5.

\section{Dynamic Model of the Robot}

Consider a rolling robot with a fluid-actuation system moving a spherical mass (core) within a circular pipe, as shown in Fig. 1. The driving mechanism pushes the fluid in the pipe to move the core. The motion of the core creates a driving moment propelling the whole spherical body. The driving mechanism is realized as a linear actuator connected through a cylinder [11].

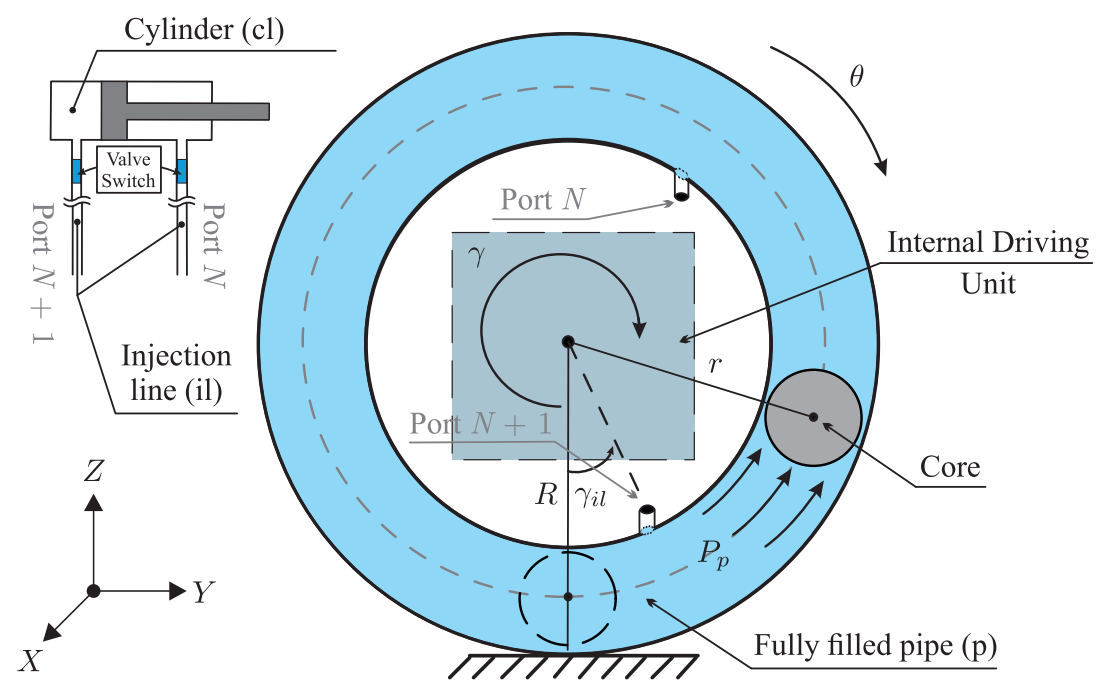

Fig. 1. Rolling motion model along the $Y$ axis with a core in a circular pipe. 
The rolling body consists of a rotating mass-point and a spherical shell, and the fluid effects on the moving core are modeled by head-loss and drag forces. Let $\theta$ be the rotation angle of the sphere, and let $\gamma$ be the relative angle of the mass point. By means of the Lagrange-Euler formalism, the nonlinear dynamics of this rolling system can be established as follows [11, 19]:

$$
\begin{aligned}
& M_{11} \ddot{\theta}+M_{12} \ddot{\gamma}+N_{1}+G_{1}=0, \\
& M_{21} \ddot{\theta}+M_{22} \ddot{\gamma}+N_{2}+G_{2}=\tau_{\gamma} .
\end{aligned}
$$

The coefficients of the equations of motion are derived as follows:

$$
\begin{aligned}
& M_{11}=M_{s} R^{2}+I_{w}+I_{s}+m_{c} R^{2}-2 m_{c} R r \cos (\gamma+\theta)+m_{c} r^{2}, \\
& M_{12}=M_{21}=I_{w}+m_{c} r^{2}-m_{c} R r \cos (\gamma+\theta), \\
& M_{22}=I_{w}+m_{c} r^{2}, N_{1}=m_{c} R r(\dot{\gamma}+\dot{\theta})^{2} \sin (\gamma+\theta), \\
& N_{2}=-0.5 r^{2} \operatorname{sgn}(\dot{\gamma})\left(f_{p} \frac{L_{p}^{\prime}}{D_{c} g}+C_{D} \rho_{f} A_{c}\right) \dot{\gamma}^{2}, G_{1}=G_{2}=m_{c}^{\prime} r g \sin (\gamma+\theta),
\end{aligned}
$$

where $r$ is the distance from the center of the sphere to the core, $R$ is the radius of the sphere, $m_{c}$ is the mass of the core, $M_{s}$ is the mass of the whole system excluding the core and the fluid, $m_{w}=\rho_{f}\left(r \pi^{2} D_{c}^{2} / 2-\nu_{c}\right)$ is the mass of the water within the pipe, $m_{c}^{\prime}=m_{c}-\rho_{f} \nu_{c}$ is the apparent mass of the core, $I_{s}=2 M_{s} R^{2} / 3$ is the inertia of the spherical shell, $I_{w}=m_{w}\left[\left(3 D_{c}^{2} / 4\right)+4 r^{2}\right] / 4$ is the rotational inertia of the water filling the pipe, $f_{p}$ is the Darcy factor, $L_{p}^{\prime}$ is the apparent length of the circular pipe, $D_{c}$ is the diameter of the core, $g$ is the gravity acceleration, $C_{D}$ is the drag coefficient, $\rho_{f}$ is the fluid density, $A_{c}$ is the cross-section area of the core, and $\nu_{c}$ is the volume of the core.

The input torque $\tau_{\gamma}$, driving the mass point, is $\tau_{\gamma}=r P_{p} A_{c}$, where $P_{p}$ is the fluid pressure in the main pipe. Note that the model contains the drag and head-loss $\left(N_{1}\right)$ as well as the buoyancy force in the apparent mass of the core $\left(m^{\prime}\right)$. We define the apparent length as $L_{p}^{\prime}=L_{p}+\left[\left(K_{b} D_{c}\right) / f_{p}\right]$ where $L_{p}$ and $K_{b}$ are the length of the circular pipe and the resistance coefficient for $180^{\circ}$ in return bend.

The input fluid pressure to the pipe, $P_{p}$, can be found with the use of the Bernoulli equation. It is obtained as $[11,20]$

$$
P_{p}=P_{c l}+\frac{\rho_{f}}{2}\left[V_{c l}^{2}-V_{p}^{2}\right]+\rho_{f} g\left[Z_{i l}-Z_{p}\left(1-\cos \gamma_{i l}\right)-h_{p}\right]
$$

where $P_{c l}, V_{c l}=A_{i l} / A_{c l} V_{i l}, V_{p}=V_{i l} A_{i l} / A_{c}, Z_{i l}, Z_{p}, \gamma_{i l}$ and $h_{p}$ are the pressure and velocity of the fluid in the cylinder, the velocity of entering fluid to the main pipe, the ground distance of the injection line and the main pipe's output port, the angle of the injection line's port and the head-loss of entering fluid to the main pipe, respectively. The head-loss $h_{p}$ is given as

$$
h_{p}=\frac{V_{i l}^{2}}{2 g}\left(f_{i l} \frac{L_{i l}}{D_{i l}}+K_{s e}\right)
$$

where $V_{i l}, f_{i l}, L_{i l}, D_{i l}, K_{s e}$ are the fluid velocity, the Darcy factor, the pipe length and the diameter of the injection line and the loss coefficient for the transition from the injection to the main pipe, respectively. The Darcy friction factors for each of the pipe sections are defined by

$$
f_{p}=64 / R e_{p}, f_{i l}=0.316 / R e_{i l}^{0.25},
$$


where Re is the Reynolds number. Each of the streamlines has its own Reynolds number as $R e=L V \rho_{f} / \mu_{f}$ where $\mu_{f}$ is the viscosity of rotating fluid. For instance, the Reynolds number for the pipe with the core $p$ is $R e_{p}=L_{p} V_{p} \rho_{f} / \mu_{f}$.

Finally, the linear actuator model is taken into consideration. It is expressed as $[11,21]$

$$
\begin{aligned}
& \dot{X}_{l}=V_{l}, \\
& \dot{V}_{l}=\frac{2 \pi \eta}{m_{l} \ell\left(1+\mu_{l}\right)} T_{m},
\end{aligned}
$$

where $X_{l}, V_{l}, \eta, m_{l}, l, \mu_{l}, T_{m}$ are the displacement and velocity of the linear actuator's rod, the efficiency of linear actuator, the mass of the rod and the connected joint, the lead gap, the friction coefficient between the rotating body and the rod, and also the DC motor torque input, respectively. Since the linear actuator changes its cycle in each arrival to the endpoint of the cylinder tank, the cross-section area of cylinder $A_{c l}$ changes by

$$
A_{c l}= \begin{cases}\pi D_{2}^{2} / 4, & \text { forward actuation } \\ \pi\left(D_{2}^{2}-D_{1}^{2}\right) / 4, & \text { reverse actuation }\end{cases}
$$

where $D_{1}, D_{2}$ are, respectively, the rod diameter and the pusher diameter in the cylinder. Note that forward/reverse actuation only exists due to the effecting area of the rod with the diameter of $D_{1}$ in the one side of the cylinder tank (see Fig. 1).

\section{Inverse Dynamics-Based Motion Planning}

In this section, we consider an inverse-dynamics based motion planning for the rest-to-rest movement of the robot. The planning problem can be formulated for two cases. In one case, the motion of the core $(\gamma(t), \dot{\gamma}(t), \ddot{\gamma}(t))$ is given, and in the other case, the motion of the sphere $(\theta(t), \dot{\theta}(t), \ddot{\theta}(t))$ is specified by time functions.

In the first case, the first equation in (2.1) is rearranged as

$$
\ddot{\theta}=-\frac{1}{M_{11}}\left(M_{12} \ddot{\gamma}+N_{1}+G_{1}\right),
$$

and the second equation in (2.1) is used for establishing the driving torque. The singularity (similar to the one established for pendulum driven rolling robots in $[22,23]$ ) may appear in (3.1) when $M_{11}$ becomes zero. It can be shown that

$$
M_{11}=\left(M_{s} R^{2}+I_{w}+I_{s}\right)+m_{c}\left(R^{2}-2 R r \cos (\gamma+\theta)+r^{2}\right),
$$

and the condition $M_{11}>0$ always holds true since

$$
M_{s} R^{2}+I_{w}+I_{s}>0,
$$

and

$$
R^{2}-2 R r \cos (\gamma+\theta)+r^{2} \geqslant(R-r)^{2} \geqslant 0 .
$$

Similar to what has been developed in [13], we presume that the core follows a two-phase motion profile shown in Fig. 2. In the first phase of this scenario, the core moves counterclockwise 


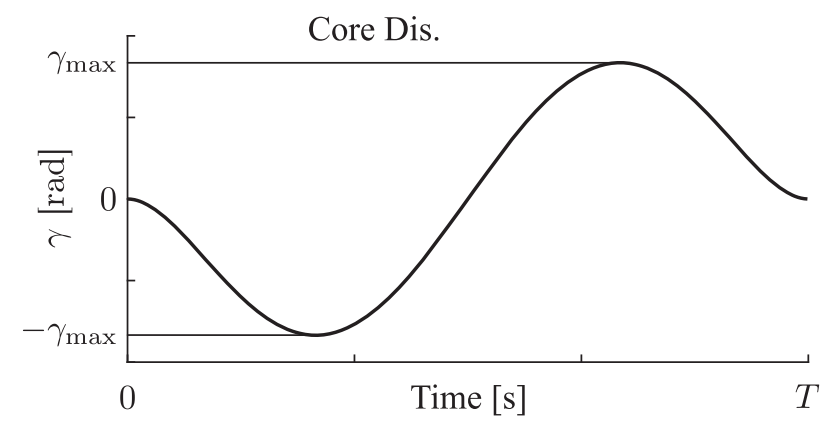

Fig. 2. Two-phase motion of the rotating core using the defined Beta function.

to $-\gamma_{\max }$, and in the second step, it returns to zero states moving clockwise. This motion profile can be captured by the 2nd order derivative of the 4 th order Beta function, that is,

$$
\gamma(t)=a\left(-\frac{840}{T^{7}} t^{5}+\frac{2100}{T^{6}} t^{4}-\frac{1680}{T^{5}} t^{3}+\frac{420}{T^{4}} t^{2}\right)
$$

where $T$ is the duration of the given movement, and the parameter $a$ is defined for the condition that $\theta(T)=\theta_{\text {des }}$, where $\theta_{\text {des }}$ is the desired displacement of the sphere. Similar to what has been established in [13], one can show that with the selection of this motion profile the condition $\dot{\theta}(T)=0$ is always satisfied.

In the second case, when the motion of the sphere is specified by a time function, the first equation in (2.1) is rearranged as

$$
\ddot{\gamma}=-\frac{1}{M_{12}}\left(M_{11} \ddot{\theta}+N_{1}+G_{1}\right)
$$

and the second equation in (2.1) is used for establishing the driving torque.

It can be shown that the condition $M_{12}>0$, that is,

$$
I_{w}+m_{c} r^{2}-m_{c} R r \cos (\gamma+\theta)=m_{w} r^{2}+\frac{3}{16} m_{w} D_{c}^{2}+m_{c} r^{2}-m_{c} R r \cos (\gamma+\theta)>0,
$$

is satisfied for any $\gamma$ and $\theta$ if

$$
\frac{m_{w}}{m_{c}}\left(1+\frac{3 D_{c}^{2}}{16 r^{2}}\right)+1>\frac{R}{r}
$$

Note that neglecting the mass of fluid $\left(m_{w}=0\right)$ leads to $\frac{R}{r}<1$, which does not hold true and thus implies the existence of singular configurations when $M_{12}$ becomes zero. However, the proper selection of the mass of the fluid so that condition (3.5) is satisfied removes the singularity from consideration.

The motion of the sphere in the second case can be specified with the use of the Beta function of the 4-th order, that is,

$$
\theta(t)=a\left(-\frac{20}{T^{7}} t^{7}+\frac{70}{T^{6}} t^{6}-\frac{84}{T^{5}} t^{5}+\frac{35}{T^{4}} t^{4}\right)
$$

where $a=\theta(T)$.

Having established the motion of the robot, $\theta(t)$ and $\gamma(t)$ (by integrating (3.1) or (3.3), depending on the chosen case) and having computed the driving torque $\tau_{\gamma}(t)$, one can then 
define the fluid pressure $P_{p}$ in the circular pipe. Note that the driving torque $\tau_{\gamma}$ is related to $P_{p}$ as $[11]$

$$
\tau_{\gamma}=r P_{p} A_{c} .
$$

Therefore, upon substituting the second equation in (2.1) with the terms from (2.2) (apparent length $L_{p}^{\prime}$ and Darcy friction $f_{p}$ ) into (3.7), one obtains

$$
\begin{aligned}
P_{p}= & \frac{1}{r A_{c}}\left(M_{21} \ddot{\theta}+M_{22} \ddot{\gamma}+N_{2}+G_{2}\right) \\
= & \frac{1}{r A_{c}}\left[\left(m_{c} r^{2}-m_{c} R r \cos (\gamma+\theta)\right) \ddot{\theta}\right. \\
& \left.+m_{c} r^{2} \ddot{\gamma}-0.5 r^{2} \operatorname{sgn}(\dot{\gamma})\left(\left(n_{1} / V_{l}\right)+n_{2}\right) \dot{\gamma}^{2}+\left(m_{c}-\rho_{f} \nu_{c}\right) g r \sin (\gamma+\theta)\right],
\end{aligned}
$$

where the constant terms $n_{1}$ and $n_{2}$ are defined as follows:

$$
n_{1}=\frac{64 \mu_{f} A_{c}}{D_{c} g \rho_{f} A_{c l}}, \quad n_{2}=K_{b} / g+C_{D} \rho_{f} A_{c} .
$$

Note that the fluid pressure $P_{p}$ depends not only on $\theta$ and $\gamma$ and their derivatives, but also on the velocity of the linear actuator $V_{l}$.

Having the defined fluid pressure $P_{p}$, one can then define the DC motor torque input $T_{m}$ with the use of the Bernoulli equation (2.3). Note that the pressure in the cylinder tank, $P_{c l}$, is related to the motor torque $T_{m}$ as [11] $P_{c l}=F_{c l} / A_{c l}=\left(2 \pi \eta / A_{c l}\right) T_{m}$. Thus, upon rearranging Eq. (2.3) with inclusion of the head-loss (2.4), one obtains

$$
T_{m}=\frac{1}{m_{1}}\left[P_{p}-m_{2} V_{l}^{2}+m_{3} V_{l}^{\frac{7}{4}}-\rho_{f} g\left(Z_{i l}-Z_{p}\left(1-\cos \gamma_{i l}\right)\right)\right],
$$

where

$$
\begin{aligned}
& m_{1}=\frac{2 \pi \eta}{l A_{c l}}, m_{2}=\frac{\rho_{f}}{2}\left[1-\left(A_{c l} / A_{c}\right)^{2}-\left(A_{c l}^{2} K_{s e} / A_{i l}^{2}\right)\right], \\
& m_{3}=\left[0.158\left(L_{i l}^{3} \rho_{f}^{3} \mu_{f} A_{c l}^{7}\right)^{\frac{1}{4}}\right] /\left(A_{i l}^{\frac{7}{4}} D_{i l}\right) .
\end{aligned}
$$

Once the expression of the motor torque is established, the states of the linear actuator, $X_{l}$ and $V_{l}$, can be defined by integrating system (2.5).

\section{Simulation Results}

In this section, the two control strategies outlined in Section 3 are tested under simulation. In the simulations, the time duration is set as $T=6 \mathrm{~s}$. The initial values for $\gamma(0), \dot{\gamma}(0), \theta(0)$, $\dot{\theta}(0), X_{l}(0)$, and $V_{l}(0)$ are zero. The desired position of the sphere is set as $\theta(T)=3 \mathrm{rad}$ and $\dot{\theta}(T)=0 \mathrm{rad} / \mathrm{s}$. The desired position of the core is set as $\gamma(T)=0 \mathrm{rad}$ and $\dot{\gamma}(T)=0 \mathrm{rad} / \mathrm{s}$. The parameters of the rolling robot are listed in Table 1 . The integration of the equations of motion is done with the use of Matlab ODE45 function, in which the integration step is set as $0.006 \mathrm{~s}$ and the relative accuracy is set as 0.001 . In the simulations, the velocity of the linear actuator, $V_{L}$, is reset to zero every time the rod reaches the end of the cylinder. Note that the sign change 
Table 1. Parameters of the robot

\begin{tabular}{c|cccccccc}
\hline Variable & $K_{s e}$ & $K_{b}$ & $\ell$ & $\mu_{l}$ & $m_{l}$ & $\eta$ & $C_{D}$ & $M_{s}$ \\
Value & 0.9 & 0.2 & $0.007 \mathrm{~m}$ & 0.75 & $0.2 \mathrm{~kg}$ & $96 \%$ & 0.8 & $1 \mathrm{~kg}$ \\
\hline Variable & $r$ & $L_{T}$ & $\mu_{f}$ & $\gamma_{i l}$ & $I_{s}$ & $D_{c}$ & $D_{i l}$ & $D_{1}$ \\
Value & $0.131 \mathrm{~m}$ & $0.05 \mathrm{~m}$ & $1.81 \times 10^{-3} \mathrm{~kg} / \mathrm{m} \cdot \mathrm{s}$ & $10^{o}$ & $0.0140 \mathrm{~kg} \cdot \mathrm{m}^{2}$ & $0.028 \mathrm{~m} 0.00635 \mathrm{~m}$ & $0.0047 \mathrm{~m}$ \\
\hline Variable & $m_{c}$ & $\rho_{f}$ & $g$ & $R$ & $D_{2}$ & $L_{p}$ & $L_{i l}$ & $I_{w}$ \\
Value & $0.25 \mathrm{~kg}$ & $1000 \mathrm{~kg} / \mathrm{m}^{3}$ & $9.8 \mathrm{~m} / \mathrm{s}^{2}$ & $0.145 \mathrm{~m}$ & $0.0097 \mathrm{~m}$ & $0.411 \mathrm{~m}$ & $0.145 \mathrm{~m}$ & $0.0086 \mathrm{~kg} \cdot \mathrm{m}^{2}$ \\
\hline
\end{tabular}

of $V_{L}$ presents the flow direction inside the pipes as it comes from solving (3.9) with $P_{p}$ and $V_{l}$ as variables. When the fluid is sucked from the input port (it is connected to the circular pipe) by the cylinder, the states $\left\{X_{l}, V_{l}\right\}$ have negative values. But when the fluid is injected from the cylinder to the circular pipe, they have positive values. In physical systems, the direction of the flow is controlled by control valves (see $[10,11]$ for the control valve integration).

In the first case, when the motion of the core is specified by (3.2), one can find $a=-15.92$ rad from the condition $\theta(T)=3 \mathrm{rad}$. The simulation results are presented in Figs. 3-4. We can observe that the given motion of the core results in the desired rest-rest displacement of the sphere. As the core moves to the upper hemisphere, its circulation begins to dissipate the positive velocity of the sphere. In particular, after $2.5 \mathrm{~s}$, where the core passes the zenith point of the sphere (see Fig. 4), the rest of its rotation creates a negative sphere velocity as opposed to the positive one (see Fig. 3 for $\dot{\theta}$ ). The dynamics of the linear actuator states are shown in Fig. 3. The desired propulsion of the sphere is successfully created by the driving mechanism. However, it should be noted that the dynamics of the linear actuator are not at rest at the final moment of time.

Core Dis.

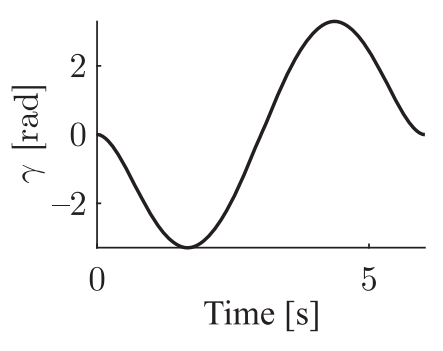

Sphere Vel.

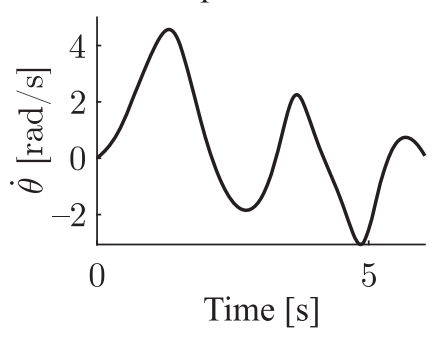

Core Vel.

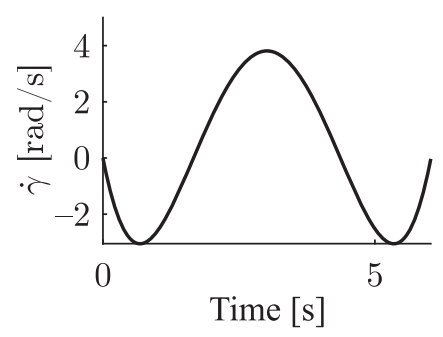

LA Vel.

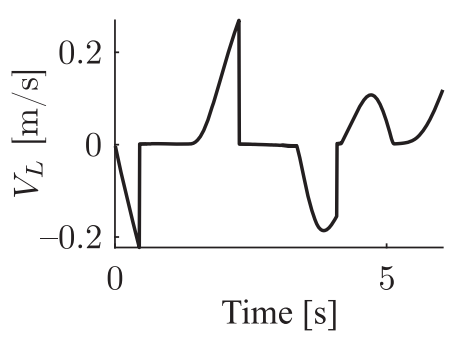

Sphere Dis.

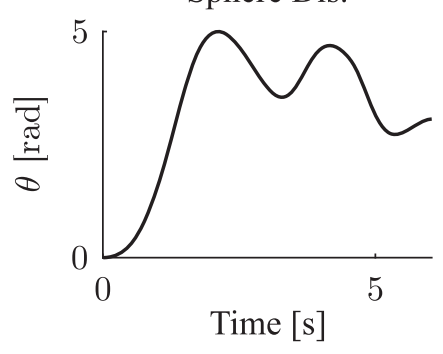

LA Dis.

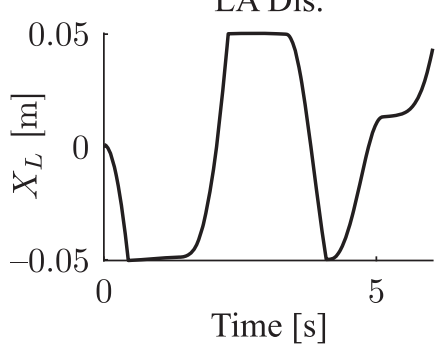

Fig. 3. Results of inverse dynamics in simulation of the first case. 

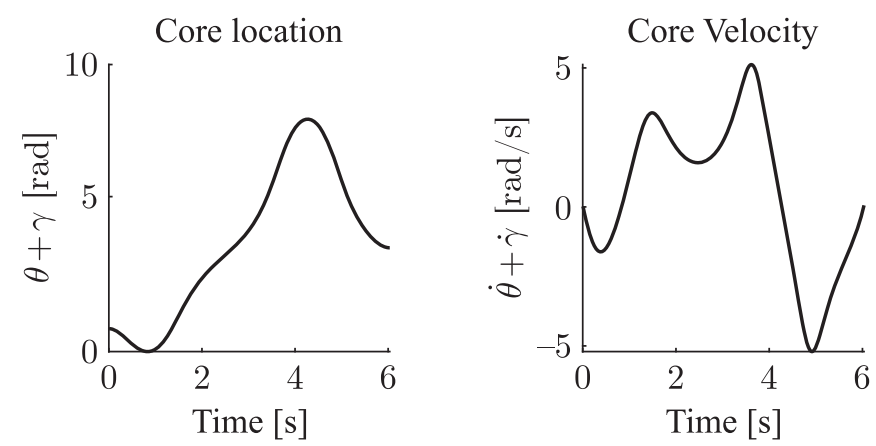

Fig. 4. Core location and velocity with respect to the base frame in the first case.

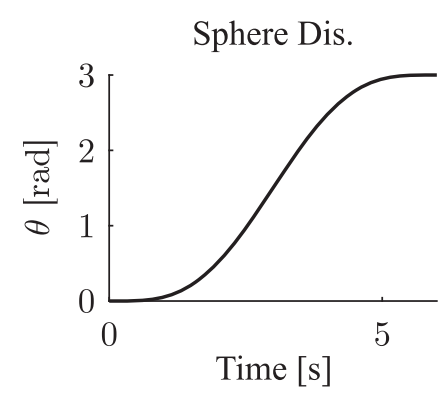

Core Vel.

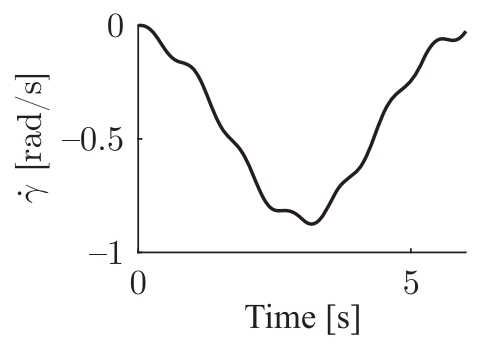

Sphere Vel.

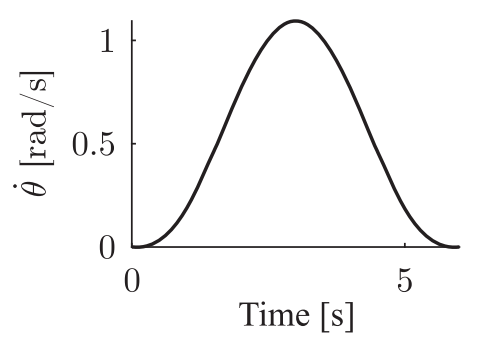

LA Vel.

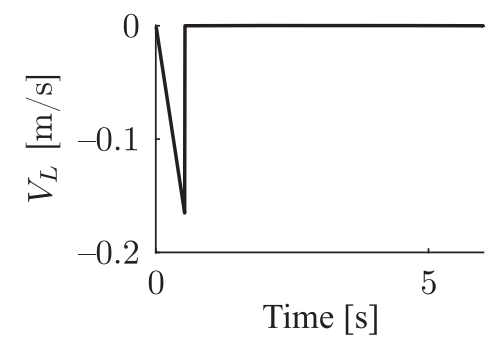

Core Dis.

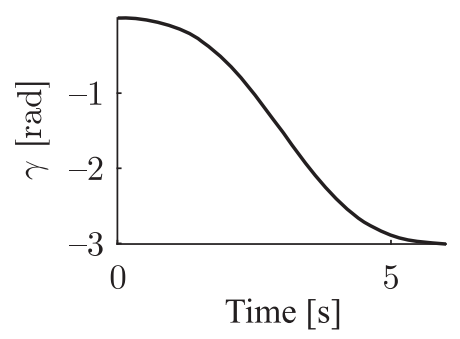

LA Dis.

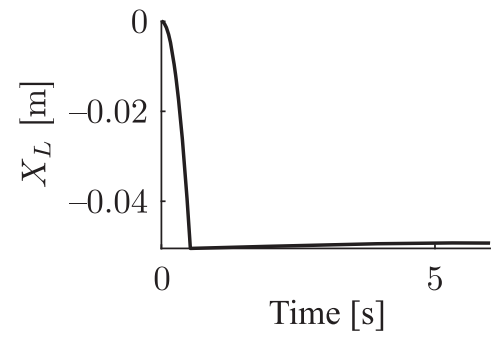

Fig. 5. Results of inverse dynamics in simulation of the second case.

In the second case, the motion of the sphere is specified by (3.6), where the parameter $a$ is set to $3 \mathrm{rad}$. The motion of the core is obtained by integrating (3.3). The simulation results are shown in Figs. 5-6. The absolute position of the core $(\gamma+\theta)$ follows, qualitatively, the two-phase motion pattern as in the first case, but with minor oscillations. However, the core $(\gamma)$ stays at the lower hemisphere (see Fig. 6). Again, one can notice that the dynamics of the linear actuator are not at rest at the final moment of time. However, it is more important to note that the velocity of the core is not exactly zero (although very close to it). Contrary to the first case where the rest-to-rest motion is ensured (the formal proof can be constructed similarly to what has been done in $[24,25]$ for the pendulum-actuated robot), the convergence of $\dot{\gamma}$ to zero cannot be guaranteed. The choice of a higher-order Beta function for the motion of the sphere may decrease the end-point velocity of the core. A systematic analysis of the convergence of $\dot{\gamma}$ to zero will be addressed in the future work.

To evaluate the performance of the control strategies, we now simulate the motion of the robot for different time constants $T \in[1.5,3]$ and desired sphere position $\theta_{\text {des }} \in[0,10]$. The purpose of these simulations is to evaluate the level of oscillations in the velocity profiles of the 

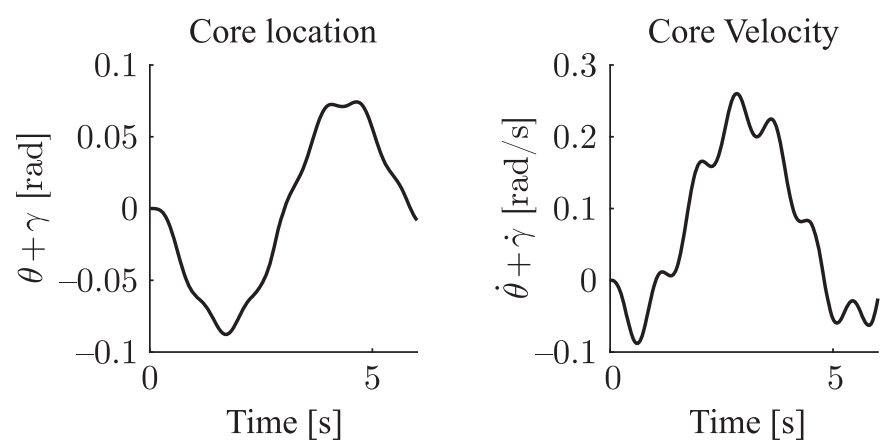

Fig. 6. Core location and velocity with respect to the base frame in the second case.

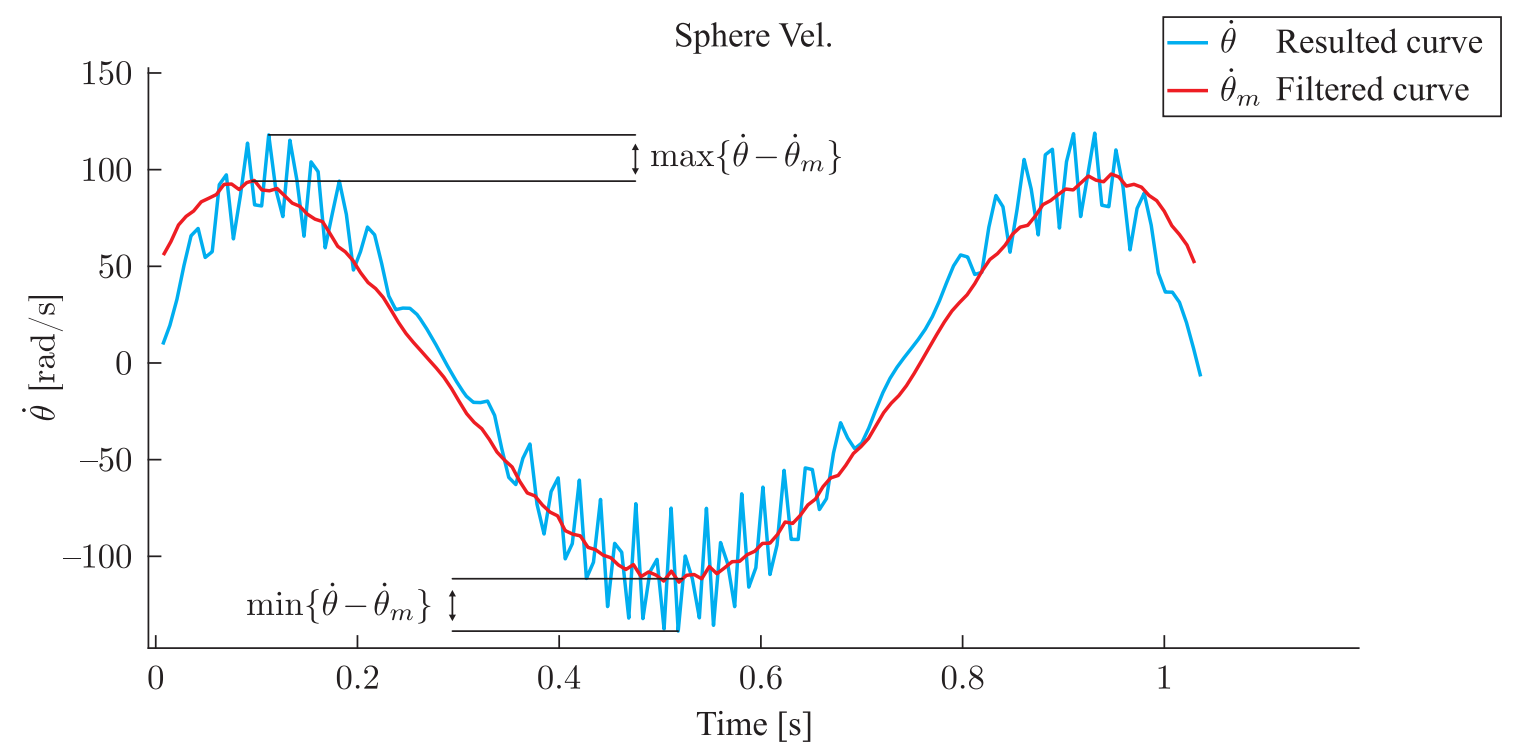

Fig. 7. Example of simulation to compute the maximum amplitude change $\Delta \dot{\theta}$ for the case that the core states are specified.

core or the sphere depending on the selected control strategy. To unify this analysis into one scheme, define $x$ to be the variable defined by numerical integration (the variable that is not specified directly by a given function of time). So, $x$ stands for either $\theta$ (obtained by solving Eq. (3.1) in the 1st control strategy) or $\gamma$ (obtained by solving Eq. (3.3) in the 2nd control strategy).

In what follows, we define the maximum amplitude change $\Delta \dot{x}$ of the velocity signal via comparing the value $\dot{x}(t)$ with the filtered curve (refer to Fig. 7 as an example of $x=\theta$ ) as follows:

$$
\Delta \dot{x}=\left|\max _{t \in[0, T]}\left\{\dot{x}(t)-\dot{x}_{m}(t)\right\}+\min _{t \in[0, T]}\left\{\dot{x}(t)-\dot{x}_{m}(t)\right\}\right| / 2,
$$

where $\dot{x}_{m}$ is the filtered curve of $\dot{x}$ found as the backward-looking moving average by

$$
\dot{x}_{m}(t)=\frac{1}{w} \int_{t-w}^{t} \dot{x}(\tau) d \tau,
$$

where $w$ is the moving average window size set as $0.1 \mathrm{~s}$ in our simulations. 
(a)

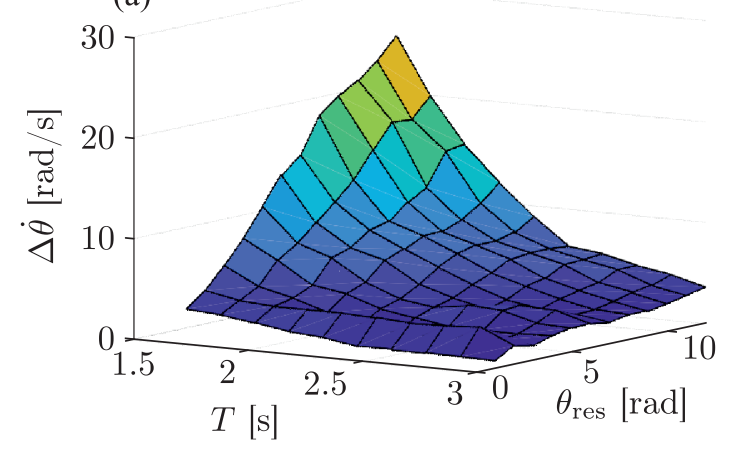

(b)

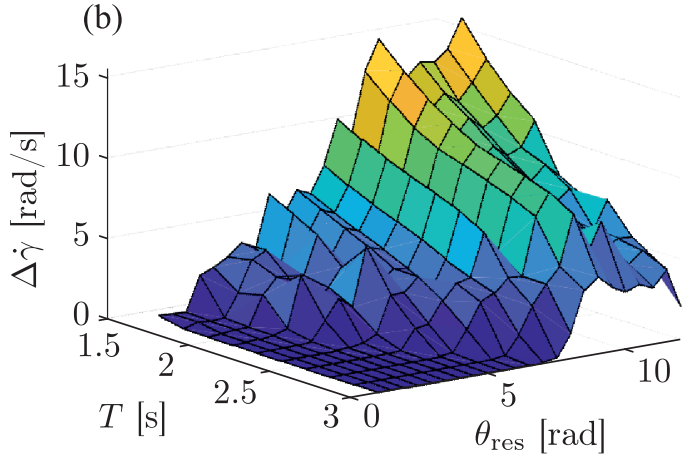

Fig. 8. a) Velocity fluctuation of the carrier state when the core states are given. b) Velocity fluctuation of the core when the carrier states are given.

The simulation results are shown in Fig. 8. For the case when the motion of the core is given (see Fig. 8a), the sphere velocity does not have serious oscillations. However, as the movement duration $T$ decreases and the traveling distance $\theta_{\text {des }}$ increases, the sphere velocity has larger oscillations. This feature also holds true for the case when the motion of the sphere is given (see Fig. 8b), however, the motion profile is more oscillating compared to the first movement strategy. As an exception, note that the peak value of the given core case $\Delta \dot{\theta}$ is very high in contrast to the given sphere case $\Delta \dot{\gamma}$ at $T \leqslant 2 \mathrm{~s}$ and $\theta_{\text {des }}>8 \mathrm{rad}$. Thus, if the motion of the core is assigned directly $(T>2 \mathrm{~s})$, the amplitude of the velocity oscillations is less compared to the case when the motion of the sphere is assigned. Overall, we can interpret that the control based on the Beta function (or any other continuous function used in specifying the desired motion) would not be suitable for a relatively short movement duration and a relatively long traveling distance.

\section{Conclusions}

In this paper, the rest-to-rest motion planning problem was addressed for a fluid-actuated spherical robot. The robot was composed of an outer shell actuated by an internal mass. The internal mass moved within a fluid-filled circular pipe fixed inside the shell. The motion of the mass was led by the fluid circulation in the pipe created by a linear actuator.

To develop feasible control laws, the dynamic model of the spherical rolling robot including the driving mechanism was first derived and then, two feed-forward control methods were proposed. The methods were based on the parameterization for the motions of the shell or that of the internal mass by Beta functions. Finally, the validity of the proposed feed-forward methods was tested under simulations.

It should be noted that in this paper we dealt only with the planar motion of the rolling robot. The development of control algorithms for three-dimensional motion will be addressed in a separate paper. Also, experiments will be conducted in future work.

\section{References}

[1] Armour, Rh. H. and Vincent, J.F., Rolling in Nature and Robotics: A Review, J. Bionic Eng., 2006, vol. 3, no. 4, pp. 195-208.

[2] Halme, A., Schonberg, T., and Wang, Y., Motion Control of a Spherical Mobile Robot, in Proc. of the 4th Internat. Workshop on Advanced Motion Control (Mie, Japan, 1996): Vol. 1, pp. 259-264. 
[3] Bicchi, A., Balluchi, A., Prattichizzo, D., and Gorelli, A., Introducing the "SPHERICLE": An Experimental Testbed for Research and Teaching in Nonholonomy, in Proc. of the IEEE Internat. Conf. on Robotics and Automation (Albuquerque, N.M., USA, 1997): Vol.3, pp. 2620-2625.

[4] Karavaev, Yu. L. and Kilin, A. A., The Dynamics and Control of a Spherical Robot with an Internal Omniwheel Platform, Regul. Chaotic Dyn., 2015, vol. 20, no. 2, pp. 134-152.

[5] Chowdhury, A. R., Soh, G.S., Foong, S., and Wood, K. L., Implementation of Caterpillar Inspired Rolling Gait and Nonlinear Control Strategy in a Spherical Robot, J. Bionic Eng., 2018, vol. 15, no. 2, pp. 313-328.

[6] Javadi, A. H. and Mojabi, P., Introducing August: A Novel Strategy for an Omnidirectional Spherical Rolling Robot, in Proc. of the 2002 IEEE Internat. Conf. on Robotics \& Automation (Washington, D.C., May 2002): Vol. 4, pp. 3527-3533.

[7] Behar, A., Matthews, J., Carsey, F., and Jones, J., NASA/JPL Tumbleweed Polar Rover, in Proc. of the 2004 IEEE Aerospace Conf. (Big Sky, Mont., March 2004): Vol. 1, pp. 395-407.

[8] Liu, D., Sun, H., and Jia, Q., Stabilization and Path Following of a Spherical Robot, in Proc. of the IEEE Conf. on Robotics, Automation and Mechatronics (Chengdu, China, Sept 2008), pp.676-682.

[9] Mahboubi, S., Seyyed Fakhrabadi, M. M., and Ghanbari, A., Design and Implementation of a Novel Spherical Mobile Robot, J. Intell. Robot. Syst., 2013, vol. 71, no. 1, pp. 43-64.

[10] Tafrishi, S. A., "RollRoller" Novel Spherical Mobile Robot Basic Dynamical Analysis and Motion Simulations, Master's Dissertation, Sheffield, Univ. of Sheffield, 2014, 81 pp.

[11] Tafrishi, S. A., Svinin, M., Esmaeilzadeh, E., and Yamamoto, M., Design, Modeling, and Motion Analysis of a Novel Fluid Actuated Spherical Rolling Robot, ASME J. Mech. Robot., 2019, vol. 11, no. 4, 041010, 10 pp.

[12] Kayacan, E., Kayacan, E., Ramon, H., and Saeys, W., Adaptive Neuro-Fuzzy Control of a Spherical Rolling Robot Using Sliding-Mode-Control-Theory-Based Online Learning Algorithm, IEEE Trans. Cybern., 2013, vol.43, no. 1, pp. 170-179.

[13] Svinin, M., Bai, Y., and Yamamoto, M., Dynamic Model and Motion Planning for a PendulumActuated Spherical Rolling Robot, in Proc. of the 2015 IEEE Internat. Conf. on Robotics and Automation (ICRA), pp. 656-661.

[14] Brown, H. B. Jr. and Xu, Y., A Single-Wheel, Gyroscopically Stabilized Robot, in IEEE Internat. Conf. on Robotics and Automation (Minneapolis, Minn., 1996): Vol.4, pp.3658-3663.

[15] Bhattacharya, S. and Agrawal, S. K., Spherical Rolling Robot: A Design and Motion Planning Studies, IEEE Trans. Robot. Autom., 2000, vol.16, no. 6, pp. 835-839.

[16] Borisov, A. V., Kilin, A. A., and Mamaev, I. S., How to Control Chaplygin's Sphere Using Rotors, Regul. Chaotic Dyn., 2012, vol. 17, nos. 3-4, pp. 258-272.

[17] Morinaga, A., Svinin, M., and Yamamoto, M., A Motion Planning Strategy for a Spherical Rolling Robot Driven by Two Internal Rotors, IEEE Trans. on Robotics, 2014, vol. 30, no. 4, pp. 993-1002.

[18] Schroll, G.C., Design of a Spherical Vehicle with Flywheel Momentum Storage for High Torque Capabilities, Thesis for Bachelor Degree, Cambridge, Mass., MIT, 2008, 26 pp.

[19] Tafrishi, S. A., Esmaeilzadeh, E., Svinin, M., and Yamamoto, M., A Fluid-Actuated Driving Mechanism for Rolling Robots, in Proc. of the IEEE 4th Internat. Conf. on Advanced Robotics and Mechatronics (ICARM, Toyonaka, Japan, July 2019), pp. 256-261.

[20] Street, R. L., Watters, G.Z., and Vennard, J. K., Elementary Fluid Mechanics, 7th ed., New York: Wiley, 1995.

[21] Childs, P. R., Mechanical Design, 2nd ed., Oxford: Butterworth-Heinemann, 2003.

[22] Kilin, A. A., Pivovarova, E. N., and Ivanova, T. B., Spherical Robot of Combined Type: Dynamics and Control, Regul. Chaotic Dyn., 2015, vol. 20, no. 6, pp. 716-728.

[23] Balandin, D. V., Komarov, M. A., and Osipov, G. V., A Motion Control for a Spherical Robot with Pendulum Drive, J. Comput. Sys. Sc. Int., 2013, vol.52, no.4, pp.650-663; see also: Izv. Ross. Akad. Nauk. Teor. Sist. Upr., 2013, no. 4, pp. 150-163. 
[24] Bai, Y., Svinin, M., and Yamamoto, M., Motion Planning for a Hoop-Pendulum Type of Underactuated Systems, in Proc. of the IEEE Internat. Conf. on Robotics and Automation (ICRA, Stockholm, May 2016), pp. 2739-2744.

[25] Bai, Y., Svinin, M., and Yamamoto, M., Dynamics-Based Motion Planning for a Pendulum-Actuated Spherical Rolling Robot, Regul. Chaotic Dyn., 2018, vol. 23, no. 4, pp. 372-388. 